\title{
ESTUDIOS GENERALES Y TECNOLOGÍA EN UN CURRÍCULO PARTICIPATIVO
}

\section{General Studies and Technology in a Participatory Curriculum}

\section{María Elena Córdoba}

"¿Quisiera Ud. decirme qué camino debo tomar para irme de aquí? -Eso depende en mucho del lugar a donde quiera irrespondió el Gato".

Alicia en el país de las maravillas

Resumen: La siguiente reseña recoge y enfatiza los puntos medulares presentados y discutidos en el marco del seminario titulado: "El papel de los estudios generales en las instituciones con fuerte énfasis en la ciencia y la tecnología" realizado en INTEC en 2012. Se subraya la importancia del rol del docente en la formación del individuo a través de un proceso abierto de construcción de conocimientos, así como de valores vinculados a la justicia, la libertad y los desafíos de la era de la información. En este contexto se reconoce la relevancia de los estudios generales y su conexión con conocimientos integrados mediante el currículo.

Palabras claves: Estudios generales, tecnología, educación, formación, currículo participativo, ciencia.

\footnotetext{
${ }^{1}$ En esta presentación no hacemos diferencia entre Estudios Generales y Educación General
} 
Abstract: The following review reflects and emphasizes the key points presented and discussed at the seminar entitled: "The role of institutions in general studies with a strong emphasis on Science and technology" made in INTEC in 2012. The importance of the role of teachers in shaping the individual and learning through an open process of building knowledge and valúes related to justice, freedom, and taking into account the challenges of the era of information. In this context, recognizes the importance of general studies and its connection with integrated knowledge through participatory curriculum.

Keywords: General studies, technology, education, training, curriculum participatory, Science.

En las últimas décadas, las demandas en materia educativa se han sentido con mayor urgencia que en cualquier otro momento ${ }^{2}$. Quizás las necesidades sociales, los deterioros medioambientales y los reclamos de organismos como UNESCO ("La justicia social la PaKJ la armonía con nuestro entorno natural deben ser las palabras claves de este mundo en devenir $j$ evidencian el papel fundamental de la educación como instrumento poderoso para accionar hacia un mundo mejor; todos mostraron la necesidad de dar una mirada atenta y autocrítica a todo el sistema educativo.

Nos queda mucho por transitar. Los esfuerzos realizados hasta la fecha no están siendo suficientes. Entre otras cosas, aún existen

${ }^{2}$ La siguiente reseña surge de una ponencia presentada en el marco del seminario titulado: "El papel de los estudios generales en las instituciones con fuerte énfasis en la ciencia y la tecnología". Este evento se realizó durante el 4 y 5 de octubre de 2012 en las instalaciones del Instituto Tecnológico de Santo Domingo (INTEC). 
barreras y resistencias que vencer. Por ello es necesario reconocer que se requiere una profunda revisión de los paradigmas en materia educativa para que den respuesta a las exigencias de la nueva sociedad y a los avances vertiginosos en ciencia, tecnología e innovación, para garantizar se atienden, de un modo eficiente, aquellas demandas sociales a las que hasta la fecha no se han dado respuestas adecuadas. Solo basta revisar a nivel mundial cómo las brechas de marginación han aumentado a la par con la pobreza y la desigualdad y cómo el accionar de las instituciones de Educación Superior, con frecuencia, han representado una utopía o tibios acercamientos en los anhelos de paz, justicia y equidad. Quizás en algunos casos, la atención prestada a responder estas necesidades del mercado desvirtuó el compromiso primero de las IES, que es con la sociedad.

Los observatorios, los avances en pedagogía y los hallazgos de investigaciones educativas coinciden en que, entre los aspectos que es necesario enfrentar para transitar hacia la tan anhelada educación como instrumento para la paz y el bien común, está el que por lo general se cuenta con un Currículo descontextualizado, centrado en contenidos declarativos en el que existe una evidente ruptura entre teoría y práctica y los contenidos con frecuencia resultan poco útiles, poco motivantes $\mathrm{y}$, por ende, poco significativos. "Frente a los numerosos desafíos del porvenir, la educación constituye un instrumento indispensable de paz, libertad y justicia social." (Jacques Delors, 1997).

Postula Tünnermann (2010) que la educación debe promover la formación de individuos cuya interacción creativa con la información les lleve a construir conocimientos. Esta interacción es posible en diseños curriculares que rompan la estructura tradicional de la docencia desde las teorías educativas centradas en los aprendizajes. Hoy en día es una realidad el aprendizaje mediado por las TIC, cuyas herramientas cognitivas se conjugan en tecnología multimedia, hipertexto e Internet, en un espacio de aprendizaje que puede estar diseñado como ambiente colaborativo, continuo y situado, centrado en los estudiantes. 
Al analizar los modelos educativos y diseños curriculares en diversas IES de América Latina y el Caribe, comprobamos que efectivamente están sustentados en enfoques centrados en el aprendizaje y enfatizan el rol más activo de los estudiantes, así como el paso del rol del docente tradicional hacia un mediador o facilitador en la construcción del conocimiento, capaz de aplicar estrategias más acordes a las necesidades de los estudiantes y del momento actual.

Sin embargo, en la práctica, con gran frecuencia no queda tan claro el rol igualmente importante del docente; llegando en algunas instituciones a no tener claridad sobre el papel del docente en un modelo de tal tipo. Incluso existen algunos docentes que lo evidencian, cuestionándose qué implica realmente ser "facilitador" o qué repercusiones tiene no ser nativos digitales, no estar actualizados con las TIC, etc. En el prefacio escrito por Ana M. Torres a una obra de Freire P. (2004) plantea: En realidad la educación que algunos avigoran como la educación del siglo XXI — televisión, video, computadoras y aparatos de todo tipo, modalidades a distancia, autodidactismo, enseñanza individualizada, aprendizaje programado, paquetes multimedia-tal parecería no incluir a los maestros y tener reservado para ellos, por el contrario, un proyecto de extinción.

Abrazar enfoques centrados en el aprendizaje no implica que la enseñanza desaparece, se integra en el proceso de tal forma que la construcción del conocimiento es posible tanto para estudiantes como para maestros, los cuales en el proceso, también aprenden. El rol del docente continúa siendo fundamental para el éxito educativo; y si bien el rol del estudiante cobra protagonismo y resulta mucho más activo que el que tenía en una concepción tradicional, le compete al docente proveer una ayuda que no es fortuita, sino programada y planificada con elementos disciplinares y pedagógicos que la posibiliten. De ahí que cuando en el INTEC se inició el proceso de reforma en el 2009, partimos de esta primera reflexión: la concepción del rol docente debería ser revalorizada a la luz de los cambios de paradigmas en educación que requieren movimientos y reconstrucciones para lograr fortalecer el proceso enseñanza aprendizaje, ya que para que ese aprendizaje disciplinar ocurra, el rol del docente también 
debe concebirse protagónico. De esta manera coincidimos con Tünnermann cuando considera que la enseñanza es un proceso de creación y no de simple repetición: "Enseñar es esencialmente proporcionar una ayuda ajustada a la actividad constructiva de los alumnos".

La segunda reflexión — del seminario mencionado más arriba— giró en torno de los docentes y a su modo de participar en la toma de decisiones curriculares. En general, el maestro recibe un programa de asignatura para impartirla sin conocer el perfil de egreso en el que se enmarca, tampoco conoce el diseño curricular y enfoque educativo que lo justifican. Por ende, el dominio de la disciplina no resulta suficiente como para vincular la asignatura con el resto de las que conforman el plan educativo; de tal forma que los contenidos no están integrados, sino que se imparten de manera desarticulada entre sí.

El tercer elemento de análisis subrayó la idea de qué enseñar, cuándo y cómo hacerlo. El cuestionamiento surgió al reflexionar sobre cómo es posible que el currículo se diseñe por un lado y las asignaturas por otro, sin estar vinculadas entre sí. Y en tal caso, cómo es posible definir de manera realmente integral lo que se considera fundamental que los estudiantes se lleven, independientemente, de la carrera que estudian; cuál es el momento adecuado para incorporar tales contenidos que debe ser el mismo para todas las carreras; y, finalmente, cómo deberían construirse esos saberes.

Muy puntualmente reflexionamos sobre los aportes de la Era de la Información, como prefiero nombrarla, la cual evidentemente nos aporta un cúmulo de datos; acceso a noticias en el mismo momento en que ocurren; respuesta a preguntas, guías y tutoriales de casi todos los temas que imaginemos; programas para crear, elaborar, configurar, programar; así como fuentes y bases de datos con la información más actualizada sobre todas las áreas académicas, etc. Ahora bien, ¿están los docentes preparados para el aprovechamiento de los recursos que la tecnología ofrece actualmente? ¿Podemos hablar de 
que existe alfabetismo informacional en los maestros? "Un docente que está estancado en el pasado y no tiene visión de futuro no puede invitar a madurar, porque tiene un código de personalidad y cultural que no corresponde a sus contemporáneos, que son sus alumnos." (Josefina Semillán, fragmento de una conferencia: sin paginación).

Estos temas sirvieron de base para que en el INTEC se llevara a cabo un análisis reflexivo con el fin de proponer un cambio capaz de atender, en primer lugar, a las demandas de la sociedad y los intereses de los actores involucrados en el proceso educativo, para posteriormente responder a las demandas del mercado laboral y que, evidentemente, tomara en cuenta los avances en tecnología educativa y en teorías del aprendizaje y la educación, en el marco de que somos un Instituto Tecnológico.

De esta forma consideramos como principal elemento en este proceso, la seguridad de que el éxito en cualquier modificación curricular dependería de la participación de todos los actores del hecho educativo y que tales cambios no serían diseñados en un escritorio, sino fruto del sentir de los involucrados. Así entonces, para lograr la presencia docente en la toma de decisiones curriculares, se organizaron comisiones que analizaron ciclos, carreras y asignaturas; las cuales estuvieron conformadas y dirigidas por maestros. Tal decisión pretendía además que cada docente, al asumir la responsabilidad de impartir una asignatura, hubiese participado o se le diera a conocer el currículo en el cual está inserta dicha asignatura, el perfil de egreso definido y la postura que asumimos en Desarrollo Curricular. Para lograr esta aspiración se consideró necesario, entre otras medidas, superar el modelo de asignaturas por el de un diseño modular en el que cada módulo integra contenidos y define competencias.

Este formato modular podría aplicarse en la práctica en la medida que se integre la labor de los maestros y se rediseñaran los procesos de formación docente, para lo cual se impondría escuchar sus experiencias y reflexionar sobre los paradigmas educativos en colectivo. Se propuso entonces una forma de trabajo en Comunidades de 
Práctica (CoP). Estas estarían formadas por los docentes que imparten las asignaturas correspondientes a cada módulo. En ellas se propuso reflexionar, analizar y evaluar permanentemente el currículo en la praxis $\mathrm{y}$, además, compartir experiencias entre pares, lo que genera un espacio de aprendizaje continuo. Una CoP, según Etienne Wenger, consiste en un "grupo de personas que comparten un interés, un conjunto de problemas o una pasión sobre un tema y quienes profundizan su conocimiento y experticia en el área a través de una interacción continua que fortalece sus relaciones."

"El puente entre este hoy y ese mañana que queremos tiene un nombre y se llama educación. Y miren que es un puente largo y difícil de cruzar. Pero hay que hacerlo... ahora, cuando todavía está fresco el milagro tecnológico de Internet y se abren oportunidades nunca vistas de acceso al conocimiento. Esas son las herramientas que nos habilitan a interactuar con la explosión universal del conocimiento." (José Mujica, presidente de Uruguay)

En INTEC las tecnologías de la información y la comunicación (TIC) son parte del eje central del quehacer institucional, debido a la naturaleza y filosofía que lo caracterizan, en ellas se enfatizan el papel de las personas, las metas, objetivos y política institucional, de los cuales depende el éxito en la utilización de las TIC para los programas educativos.

Por nuevas tecnologías de la información y la comunicación (NTIC) entendemos el conjunto de herramientas, técnicas y metodologías utilizadas para el tratamiento, esquematización, recuperación, presentación y transmisión de información en las más variadas formas. Son concebidas como un instrumento, que utilizado responsablemente, posibilita la libertad y la democracia de acceso a la información, así como de los medios necesarios para propagar los conocimientos y facilitar la participación en comunidades de aprendizajes, en el entendido de que las NTIC son solo una herramienta y no una solución a la problemática educativa. 
Asumir esta forma de trabajo no garantizaba que estuviéramos logrando el tránsito hacia un currículo que aprovechara al máximo los recursos de las TIC, decisión que como institución tecnológica era evidente; ni tampoco que se supere la precariedad de los procesos participativos que caracterizan la cultura latinoamericana. Por ello se consideró apropiado comenzar a transitar hacia un diseño curricular "blendecP, integrador y consensuado, como una de las formas de generar espacios para la reflexión y la participación. Desde ese momento, las áreas de Informática, Biblioteca y Currículo entrelazaron sus acciones para dotar a los docentes de las herramientas y recursos tecnológicos que promueve este Currículo: se generaron iniciativas concebidas y desarrolladas en el marco de la "Alfabetización Multimodal", una de cuyas dimensiones es la alfabetización informacional; talleres de ambientes virtuales de aprendizaje, tecnología educativa, constructivismo social y el conectivismo a través de la plataforma de Moodle constituyen los ejes de la formación docente.

Para lograr la incorporación de las NTIC, se realizan esfuerzos con el objetivo de hacer llegar a los diferentes espacios académicos, la capacitación en el uso de estas tecnologías, de acuerdo a los lincamientos pedagógicos y al diseño curricular vigente, así como en la evaluación de los resultados a través del seguimiento y sistematización del proceso.

Estos espacios de capacitación se ofrecen en CoP con el objetivo de reflexionar en colectivo sobre las mejores prácticas y utilización responsable de las NTIC en el ámbito educativo. Para ello, se fomentan espacios de reflexión, producción de materiales e intercambios de experiencias con otras universidades de América Latina y el Caribe a fin de analizar los requerimientos de la sociedad en general y de la educación en particular; así como también las ventajas y riesgos del uso de las tecnologías en los procesos educativos, que pueden servir tanto para fortalecer los aprendizajes como para desvirtuarlos. Esto se hace con el fin de apoyar la construcción de un modelo que tienda a la paz o como instrumento de poder para perpetuar el sistema imperante, todo depende de quién las esté utilizando y con 
qué fines, por ello los docentes tienen que estar prevenidos de tales desenlaces y de allí que resulte absolutamente necesario enmarcar las TIC en una estructura que favorezca la reflexión y el análisis crítico, como ocurre con las posibilidades que brindan los Estudios Generales, en cuya esencia está el desarrollo del espíritu crítico y reflexivo del hecho educativo. En resumen, para que las tecnologías tengan un impacto positivo en la educación, deben visualizarse como soporte a la docencia, y como recurso mediador que facilite el aprendizaje activo y no como factor determinante en el proceso.

Con esta concepción del uso de las tecnologías, la lógica para incorporar un diseño tipo "blended" reside en el uso flexible del tiempo, el aprovechamiento de ambientes virtuales de aprendizaje, mayor participación entre estudiantes y con el docente y una modalidad mucho más motivante para los estudiantes gracias al empleo de las nuevas tecnologías de la información y la comunicación. Por su parte, el conectivismo está dando lugar a un accionar pedagógico que utiliza la conectividad, aprovechando los recursos que ofrece la web con el objetivo de mejorar el proceso de enseñanzaaprendizaje.

Siguiendo con la línea de pensamiento de Josefina Semillán (2010), ella plantea que si no hay diversidad se muere la identidad. Por lo cual es necesario revalorizar los aspectos éticos y culturales de la educación, donde cada uno finalmente apunte hacia la unidad de un mundo que hoy se ve totalmente caótico, sin ningún respeto por el otro. Es indispensable ponernos a la par y comprender que los jóvenes y adolescentes han trasladado sus estilos a como están formateados, porque piensan en términos de red y se comunican por puntos de afinidad y no por empatia de valores; el tiempo es simultáneo, no sucesivo.

Hay que internalizar esta revolución tecnológica e informativa, es una obligación en educación, para ello lo primero es aceptar nuestras limitaciones y analizar las formas de superarlas, a fin de lograr descifrar los nuevos códigos culturales como, por ejemplo, que los 
jóvenes en general, se relacionan con la información abarcando mucho pero superficialmente, nos compete entonces aprender formas que resulten acordes a esta generación para que seamos capaces de invitar a disfrutar y sentir el placer por el conocimiento.

La propuesta presentada fomenta la participación y dimensiona el mejor aprovechamiento de la tecnología, con integración de contenidos disciplinares y docentes. Y fue allí que cobró sentido la incorporación de los Estudios Generales en un Instituto Tecnológico; recordemos, en primer lugar, la Conferencia Mundial de Educación Superior de París, julio de 2009: "En ningún momento de la historia ha sido tan importante como ahora contar con la garantía de la calidad de los estudios superiores, por su condición de fuerza primordial para la constmcción de sociedades del conocimiento, integradoras y diversas y para fomentar la investigación, la innovación, la creatividad y el desarrollo". De esa forma integradora del conocimiento, capaz de atender a necesidades reales y que revalorice los aspectos éticos y culturales, es que entendemos el espíritu de los Estudios Generales, donde "educar es decodificar los nuevos lenguajes para responder a necesidades reales y no pretéritas. Es invitar a estar entusiasmados, la motivación es reinauguración del lenguaje y esa pasión se logra en conjunto con los demás docentes." (Semillán, 2010: sin paginación). En CoP que reflexionan sobre la humanización y valores que promueven los Estudios Generales es posible lograr esa pasión:

Ea educación general concibe el saber como gestación continua que implica renovación, búsqueda, problemati?(ación, ponderación reflexiva, elaboración conceptual, investigación, creación y comunicación. Su orientación pedagógica es la promoción de una experiencia que acentúe el proceso formativo de contenidos cognoscitivos, habilidades y sensibilidades reflexivas, creativas y críticas. Dos ejes articulan la educación general: el planteamiento sobre el carácter abierto y cambiante de las visiones de mundo y la integración como principio básico del conocer Mensaje de Revisión de Bachillerato (2006), Senado Académico. Recinto Río Piedras. 
Estamos conscientes de que la Educación General puede abordarse desde diversos enfoques y adquirir interpretaciones y modalidades diferentes, como plantea Vélez W.: "La educación general que necesitamos tiene que fundamentarse en la integración de los conocimientos y en el cuestionamiento de las disciplinas tradicionales que vienen "disciplinando" los saberes, sobre todo desde el siglo XIX". Allí nos posicionamos al decidir incorporar la educación general al currículo del INTEC.

El fuerte énfasis en la tecnología del INTEC se vio enriquecido a partir de la reforma curricular del 2010, al asumir un ciclo de Estudios Generales. Este ciclo se concibe como el espacio formativo que sienta las bases y proporciona los instrumentos, conceptos y modos que dan fundamento a la Formación Integral del estudiante, ya que es allí donde se sustentarán las bases de la responsabilidad consigo mismo y con la sociedad. Fue concebido en cinco módulos que integran contenidos de las áreas académicas de Ciencias Básicas y Ambientales, de Ciencias Sociales y Humanidades. El mismo representa el sello del INTEC porque abarca la educación que es común a todo estudiante inteciano $\backslash$ constituye su sello distintivo; y está enfocado hacia la formación general, la cual se continuará a lo largo de toda la carrera. Cabe señalar que no debe conceptualizarse como una instancia remedial de las deficiencias preuniversitarias sino como un espacio de aprendizaje destinado a la integración de los elementos requeridos para enfrentar la realidad, el conocimiento científico y estar preparados para un mundo en constante cambio.

Gracias a la incorporación de los Estudios Generales, pudimos comenzar a replantearnos aspectos de la integración en el entendido de que derribar las barreras tradicionales entre las disciplinas y lograr esa vinculación, reformulando y humanizando el aprovechamiento de la tecnología, lo podíamos resolver con la construcción de un pensamiento reflexivo en CoP donde el rol del docente es revalorizado $\mathrm{y}$ son los maestros quienes van marcando pautas de los contenidos curriculares de los Estudios Generales con una modalidad de enseñanza situada, construida entre docentes y con estudiantes. "El 
conocimiento es situado, cuando es parte y producto de la actividad, del contexto y la cultura en la que se desarrolla y se utiliza. El modelo de enseñanza situada se centra en prácticas educativas auténticas, significativas y propositivas." (Díaz Barriga F., 2005: 45).

El actual Currículo de INTEC aspira a la Formación Integral y se sustenta en la praxis. Específicamente la racfim de ser del Ciclo de Educación General radica en la adquisición de las competencias generales que faciliten a los estudiantes la apropiación de conceptos, habilidades, destrejas, valores y actitudes encaminados a desarrollar la inteligencia, la afectividad y el compromiso social y a adquirir puntos de referencia morales e intelectuales que fomenten la reflexión y respeto por el mundo que les rodea, a fin de reflejarlo en un accionar personal y profesional responsable y crítico. Córdoba, 2011: 2.

En la conferencia Regional de Educación Superior Latinoamericana y del Caribe de junio de 2008 se planteó que "la mundialización ha puesto de relieve la necesidad de establecer sistemas nacionales de acreditación de estudios universitarios y de garantía de calidad, así como promover la creación de redes entre estos sistemas."

Por lo antes dicho, resulta necesario asumir que la transformación hacia la conformación de redes del conocimiento con docentes participando en CoP tiene que ser reforzado aún más y contar no solo con una voluntad política a nivel institucional, sino con el compromiso responsable de funcionarios y directivos institucionales, a fin de favorecer toda oportunidad y espacio de reflexión y evaluación hasta que logremos la transformación que desde la reforma curricular se está impulsando con el objetivo de lograr un diseño participativo e integrador con docentes comprometidos y motivados. "En la medida que el maestro es capaz de asombrarse y transmitir el sentido de asombro a sus alumnos, es un buen maestro.” (Latapí, 2009).

Resultan indispensables estos espacios de reflexión entre docentes sobre los contenidos curriculares, sobre la modalidad del proceso y las estrategias exitosas, etc. de los Estudios Generales, así como la 
comprensión de que las TIC las tenemos que aprovechar para favorecer el aprendizaje, aplicando las técnicas adecuadas y que son esos espacios de reflexión los que previenen el riesgo de ser tecnocráticos. En tal caso, la evaluación auténtica de los procesos de utilización de las TIC nos aporta luz sobre los avances y requerimientos en ese sentido. Las TIC, como dijimos, constituyen la oportunidad de usar herramientas motivadoras al servicio de la docencia, cuyo uso esté sustentado sobre sólidas bases pedagógicas, aunque el sello institucional esté definido no por la utilización de tecnologías, sino por el ciclo de Estudios Generales en el cual se promueve la reflexión, la integración de contenidos, la contextualización de los aprendizajes, y se propicien aprendizajes situados y para toda la vida, con aprovechamiento de las TIC.

Los docentes que imparten docencia en el ciclo de Estudios Generales están integrándose paulatinamente en el accionar en $\mathrm{CoP}$, mediante la participación en diversas plataformas digitales en las que se analiza el impacto social de Internet y la utilización apropiada de herramientas dentro de la variedad que ofrecen las TIC, como por ejemplo Wiziq, redes sociales como Facebook, y documentos compartidos como los Google Docs, todo lo cual propicia la construcción de conocimientos en el marco del compromiso social y la incidencia en una cultura democrática donde la paz, la inclusión social y el respeto medioambiental son ejes fundamentales de este ciclo.

Quienes interpretamos como una exitosa alianza los Estudios Generales y las TIC, entendemos que es una forma de dar respuesta a las inquietudes que analizamos al principio de nuestra reforma, por lo que resulta) esperanzador y muy gratificante a dos años de puesto en vigencia el nuevo Currículo, compartir las experiencias de los docentes que más empeño están poniendo en esta integración y que han comenzado a sistematizar y documentar los resultados de las $\mathrm{CoP}$, sus inquietudes, preocupaciones, logros y hallazgos. Aspiramos a un mundo mejor, entendemos que la educación es el camino y como sabemos hacia dónde queremos ir, no resulta necesario preguntarle al Conejo de Alicia. Al menos en este momento y hasta 
que estemos en condiciones de hacer una evaluación profunda de este accionar, seguiremos defendiendo enfáticamente los Estudios Generales que, a juzgar por lo que hasta ahora hemos avanzado, fortalecen cualquier proceso que se sustente en el aprovechamiento de las TIC.

Sabemos que nos queda mucho por hacer en materia educativa $y$ resulta difícil en nuestra sociedad latinoamericana, en la que no tenemos cultura de evaluación, ser autocríticos y aceptar que estamos distantes de la calidad que en teoría proclamamos; que no todo es cuantificable en educación; que para posicionarnos en la excelencia académica tenemos que mejorar y aumentar la producción intelectual; que es necesario investigar mucho más, publicar, trabajar en colectivos docentes y ser capaces de mirar más allá, sin fronteras, para poder construir conocimientos a través de la investigación y promover un mundo de paz y equidad. El plantearnos en el INTEC un currículo que está siendo evaluado constantemente por los docentes en CoP y por los aportes de los estudiantes que se recogen en las mismas, resulta alentador, pero no para quedarnos tranquilos, sino por el contrario para cuestionarnos permanentemente, alentarnos a investigar más y no permanecer impávidos ante una realidad que si no la comprendemos, nos puede desbordar.

"Los holandeses andan en bicicleta, las usan para ir a trabajar pero también para ir a los conciertos o a los parques. Porque han llegado a un nivel en el que su felicidad cotidiana se alimenta tanto de consumos materiales como intelectuales. Así que amigos, vayan y contagien el placer por el conocimiento." (Mujica, 2011). 


\section{Referencias bibliográficas}

Carlino, P. (2005). Escribir; leer y aprender en la Universidad. Una Introducción a la alfabetización académica. Buenos Aires: Fondo de Cultura Económica.

Coll, C. (2010). Reformulación de formación docente en República Dominicana [Conferencia presentada en el encuentro de Reformulación organizado por la MESCYT]. Santo Domingo: MESCYT.

Coll, C. (2004). Redefinir lo básico en la educación básica. Cuadernos de Pedagogía, (s. v.) 339, 80-84.

Conferencia Regional de Educación Superior Latinoamericana y del Caribe (junio de 2008). Colombia: Instituto Internacional de la UNESCO para la Educación Superior en América Latina y el Caribe (UNESCO-IESALC).

Córdoba, M. (2011) De ciclo propedéutico a Educación General, Revista $U_{m b r a l}{ }^{\wedge}$ No. 4 (s. p.). Recuperado de http://ojs.uprrp.edu/index.php/ umbral/article/view /15.

Delors, J. La educación encierra un tesoro. Madrid: Santillana. Ediciones Unesco.

Díaz Barriga, F. (2005) Enseñanza situada. Vínculo entre la escuela y la vida. México: Me Graw-Hill.

Latapí, P. (2009). El derecho a la educación. Su alcance, exigibilidad y relevancia para la política educativa, Revista Mexicana de Investigación Educativa, 14 (40), 255-287.

Morín, E. (1999). Los siete saberes necesarios para la educación del futuro. París: Organización de las Naciones Unidas para la Educación, la Ciencia y la Cultura. Recuperado de http://www.unmsm. edu.pe/occaa/artículos/saberes7.pdf. 
Mujica, J. (25 de marzo, 2011) Mensaje del Presidente de Uruguay.

Semillán, J. (mayo, 2010) Educar para pertenecer, pertenecer para educar. [Conferencia inédita].

Senado Académico (2006, 26 de enero). Revisión del Bachillerato. [Conferencia]. Recinto de Río Piedras: Universidad de Puerto Rico.

Torres, A. en Freire, P. (2004) Cartas a quien pretende enseñar. (2da. reimp.) Buenos Aires: Siglo XXI Editores.

Tünnermann, C. (2010) Tendencias de la educación superior. Santiago de los Caballeros: Ediciones UAPA.

Vélez, W. (marzo de 2000). El currículo de educación general en el siglo XXI. [Ponencia presentada en el Décimo Segundo Encuentro Nacional de Educación y Pensamiento].

Wenger, E. (2003). Communities Communities of Practice: Leaming Meaning and Identitj. Cambridge: Cambridge University Press. 


\section{María Elena Córdoba}

Es educadora investigadora. Actualmente se desempeña como directora de Desarrollo Curricular en el Instituto Tecnológico de Santo Domingo. Estudió Psicología en la Universidad Nacional de Córdoba, Argentina; cursó una maestría en Educación Social y Animación Sociocultural por la Universidad de Sevilla-INTEC; posee un doctorado en Educación por la Atlantic International University.

Ha sido asesora y consultora en Desarrollo Curricular y Formación Docente de varias universidades de México. Se desempeñó como docente de Metodología de la investigación en diversos programas de maestrías en el Instituto de Estudios Universitarios de México. Ha impartido numerosos cursos y talleres sobre elaboración de tesis y Metodología de la Investigación; también ha dirigido investigaciones sociales, educativas y psicológicas en México, Argentina y República Dominicana. En el campo de la Investigación tiene trabajos sobre el rol y la función docente, evaluación, currículo, formación y actualización del profesorado.

Email: maria.cordoba@intec.edu.do

Recibido: 07/12/2012 Aprobado: 26/02/2013 
